\title{
ALGUNAS REFLEXIONES A SIETE AÑOS DE LA IMPLEMENTACIÓN DE LA PSU
}

\author{
Mladen Koljatic \\ y Mónica Silva
}

\begin{abstract}
Hace siete años se comenzó a aplicar en Chile la PSU, un nuevo sistema de pruebas para ingresar a la universidad. Estas nuevas pruebas estuvieron precedidas por altas expectativas en términos de las mejorías que producirían en el sistema de educación secundaria y las mayores oportunidades para los grupos más vulnerables. Este artículo revisa algunos resultados de la implementación de la PSU, centrando el análisis principalmente en temas de capacidad predictiva y equidad.

Los autores concluyen que no se advierte una mejoría en la capacidad predictiva de las pruebas PSU al aumentar los contenidos evaluados en éstas. Más aun, luego de controlar por nivel socioeconómico, se constata que el aumento de contenidos ha sido perjudicial para los estudiantes de grupos vulnerables, en especial para aquellos que provienen de la educación municipal técnico-profesional.
\end{abstract}

Mladen Koljatic M. Ingeniero Comercial de la P. Universidad Católica de Chile; MBA University of Michigan y Doctor en Educación (Ed. D.), con mención en educación superior, Indiana University. Profesor titular de la Escuela de Administración de la P. Universidad Católica de Chile.

Mónica Silva R. Psicóloga de la P. Universidad Católica de Chile; Master of Science y Ph. D. en Psicología de la Educación, Indiana University. Investigadora de la Escuela de Administración de la P. Universidad Católica de Chile. 
A la luz de las limitaciones en cuanto a equidad y capacidad predictiva de la PSU, se sugiere entonces revisar el uso que se da actualmente a ciertos puntajes de las pruebas obligatorias como puntos de cortes para asignación de beneficios y créditos. Finalmente, dado el rol clave que juega en nuestro país el acceso a la educación universitaria en la movilidad social, se sugieren auditorías periódicas, realizadas por expertos internacionales, independientes y altamente calificados, para garantizar que el sistema de admisión cumpla con altos estándares de calidad y equidad.

Palabras clave: administración universitaria; pruebas de admisión a la universidad; equidad.

Recibido: octubre 2010. Aceptado: noviembre 2010.

\section{$\mathrm{E}$}

diciembre del año 2003 se estrenó la primera versión de la PSU, el nuevo sistema de pruebas de admisión a la universidad. El esquema que había regido hasta ese entonces (PAA), de pruebas de razonamiento con escasa referencia a contenidos curriculares, complementadas por pruebas de conocimientos específicos (PCE) exigidas para algunas carreras, se cambió por un esquema de pruebas de lenguaje, matemática, ciencias, historia y ciencias sociales (PSU) que abarca extensos contenidos y todos los aprendizajes hasta cuarto medio.

El debut de la PSU estuvo precedido por una intensa controversia. Había quienes anticipaban que el cambio traería grandes beneficios para el sistema escolar. Se pensaba que alinear las pruebas de ingreso a la universidad con los contenidos de la educación media mejoraría los aprendizajes, daría la posibilidad de controlar a los establecimientos educacionales en función de sus resultados y se traduciría en una mejoría para la equidad ${ }^{1}$. Por otro lado, estaban aquellos que advertían que un cambio de tal naturaleza conllevaba grandes riesgos en un país con una gran desigualdad en el acceso a educación básica y media de calidad, y se preguntaban si las nuevas pruebas serían más adecuadas para detectar talento en un país como Chile².

${ }^{1}$ D. Bravo y otros, "Reformulación de las Pruebas de Selección a la Educación Superior", Proyecto Fondef, Santiago, Chile, 2000.

2 A. Fontaine, "Peligro en el SIES", 2002, pp. 25-55; B. Eyzaguirre \& C. Le Foulon, "El SIES: Un proyecto Prematuro", 2002, pp. 39-53. 
El mensaje de sus creadores era que el cambio era menor y que para tener una buena preparación para rendir las nuevas pruebas bastaría concentrarse en el trabajo escolar, lo que haría menos necesarios los preuniversitarios $^{3}$. Para aminorar el impacto de la transición, el Consejo de Rectores (CRUCH) hizo la concesión de no incluir la totalidad de los contenidos curriculares en las pruebas en su primer año de aplicación e ir aumentando gradualmente los contenidos hasta llegar a su máximo en el año 2007. Así, las pruebas fueron incorporando a lo largo de los años una mayor cantidad de contenidos hasta incluir la totalidad de los vastos contenidos mínimos obligatorios del marco curricular susceptibles de ser evaluados mediante pruebas de alternativas. Hubo una segunda concesión que consistió en reducir el número de pruebas exigidas para postular a las universidades del Consejo de Rectores. Si bien en el primer momento se planteó que todos los postulantes, independientemente de la carrera a la que aspirasen, deberían rendir las cuatro pruebas, finalmente se optó por dejar sólo las PSU de Lenguaje y de Matemática como obligatorias y a elección una tercera prueba (Ciencias o Historia y Ciencias Sociales), según fuera requerida para las carreras de interés de los postulantes.

A siete años del cambio a la PSU, persisten las dudas respecto a si el cambio fue beneficioso para el sistema educacional chileno y si éste cumplió con las expectativas de dar mayores oportunidades de acceso a los grupos más vulnerables. Este trabajo presenta algunas reflexiones sobre aspectos relevantes que no han sido suficientemente destacados con anterioridad y que tienen directa relación con la capacidad predictiva y equidad de los instrumentos. Estos comentarios están referidos al impacto de la PSU en el currículum de la enseñanza media y, en particular, en la educación municipal en su modalidad técnico-profesional, a la capacidad predictiva del instrumento, a la pertinencia de incluir el máximo de contenidos curriculares y al uso de las pruebas como instrumento para otorgar beneficios. Finalmente, se presentan algunas sugerencias acerca de cómo mejorar las pruebas.

\section{Las pruebas de admisión y su impacto en la enseñanza media}

Uno de los argumentos centrales en que se apoyó el cambio de pruebas fue su potencial efecto benéfico sobre la enseñanza media. Al

${ }^{3}$ SIES: Un Desafío para la Enseñanza. Portal EducarChile 26/4/2002. Accesible en: http://www.educarchile.cl/Portal.Base/Web/VerContenido.aspx?ID=73956. 
alinear las pruebas con el currículum de la enseñanza media, sus promotores esperaban que se generara un círculo virtuoso que se traduciría en un mejoramiento de la enseñanza media: los padres presionarían a las escuelas para que pasaran todo el currículum, para los profesores sería más fácil motivar a sus estudiantes para concentrarse en las materias y éstos tendrían la motivación para aprender lo que sus profesores les enseñaban porque "entraba en las pruebas".

Sin embargo, este círculo virtuoso derivó en el fenómeno de "pruebas guiando el currículum" en ciertos colegios, tal como se aprecia en el caso que se describe a continuación y que corresponde al de un colegio particular pagado. Luego de las primeras aplicaciones de la PSU, la dirección del colegio estimó que el rendimiento de su alumnado estaba "bajo lo esperado", por lo que se desarrolló una estrategia para revertir esta situación. Como primera medida, la dirección del establecimiento contrató los servicios de un preuniversitario de vasto prestigio para que hiciera clases al estudiantado dentro del horario regular, reemplazando horas de otras asignaturas para el entrenamiento PSU. Luego se organizaron talleres para preparar la prueba de Ciencias, los cuales sesionaban en grupos reducidos con profesores de Biología, Química y Física. Finalmente, durante los dos últimos meses del año, los cuartos medios sólo tuvieron clases en las disciplinas comprometidas en la PSU. Estos cambios se tradujeron en un salto de 70 puntos en el ranking de "Mejores Colegios en la PSU" del año siguiente.

En sí mismo, el fenómeno de "pruebas guiando el currículum" no es malo, en la medida en que hubiese consenso en que los contenidos que se están evaluando a través de las pruebas son realmente importantes de aprender y, por lo tanto, se justifica que desplacen a otras materias. Sin embargo, cuando los contenidos evaluados en las pruebas de admisión son inadecuados, ya sea porque son irrelevantes o enciclopédicos, las pruebas de admisión pueden constituirse en un problema. La dirección del colegio logró su propósito de mejorar el rendimiento del alumnado en la PSU, pero el costo involucrado fue alto y cabe preguntarse si la inversión redundó en una "mejor calidad de educación". Aun cuando no hay estudios empíricos a gran escala sobre la magnitud del fenómeno de las pruebas guiando el currículum y de su impacto en el aprendizaje, los indicadores de la calidad de la 
enseñanza de los últimos años en Chile, en particular del Simce, parecen señalar que el círculo virtuoso sobre el aprendizaje no ha ocurrido ya que no se advierte un progreso significativo en la calidad de la educación $^{4}$.

\section{La brecha que crece}

Dentro de las expectativas creadas en torno a las nuevas pruebas de admisión estaba que éstas darían mayores espacios de oportunidad a los jóvenes provenientes de los sectores más vulnerables del país. Se argumentaba que mientras más contenidos se incorporaran a las pruebas, mayor sería el impacto de la escuela y menor el del capital cultural o la asistencia a preuniversitarios, lo cual garantizaba que no crecería la brecha de rendimiento en las pruebas de admisión entre los distintos sectores sociales ${ }^{5}$.

La hipótesis de mayor equidad nunca fue contrastada antes de eliminar el antiguo sistema y los resultados del cambio a la PSU no fueron los esperados. La omisión de un período de marcha blanca para evaluar las PSU dificultó la posibilidad de estimar el impacto real del cambio, puesto que no se tenía al mismo grupo rindiendo ambos tipos de pruebas. Por este motivo fue necesario emplear técnicas alternativas para estimar el impacto de la PSU sobre los grupos vulnerables. Dos estrategias han sido utilizadas para evaluar el impacto del cambio: restricción de la comparación a grupos homogéneos (o grupos equivalentes) ${ }^{6}$ y control estadístico empleando modelos de regresión ${ }^{7}$. Ambas estrategias de análisis han arrojado resultados consistentes: el aumento de la brecha va más allá de lo que es susceptible de ser explicado por el cambio ocurrido en el perfil socioeconómico de quienes rinden la PSU a

\footnotetext{
${ }^{4}$ Según los resultados del Simce 2008 de Segundo Medio. Accesible en: http:// www.simce.cl/fileadmin/Documentos_y_archivos_SIMCE/Informes_Resultados_2008/ Informe_Nacional_2008.pdf.

${ }_{5}$ Declaración del director del proyecto SIES citada en crónica "El SIES Comenzará a Ser Aplicado a Contar del 2003”, Diario Austral 21/7/2002.

${ }^{6}$ Como lo hiciera H. Beyer en el documento "Igualdad de Oportunidades y Selección a las Universidades", 2009.

${ }^{7}$ M. Koljatic, M. Silva y R. Cofré, "Achievement Versus Aptitude in College Admissions: A Cautionary Note Based on Evidence from Chile", 2010, trabajo presentado en la Decimotercera Conferencia organizada por la Agencia Nacional para la Educación Superior de Suecia. Universidad de Umea, Suecia, junio 15-17.
} 
través de los años, contradiciendo las conclusiones del Comité Técnico Asesor (CTA) del Consejo de Rectores (CRUCH) ${ }^{8}$.

El aumento de contenidos en las pruebas PSU no ha favorecido a los alumnos de sectores más vulnerables sino que, por el contrario, los ha perjudicado. Aún más, dentro del grupo que asiste a colegios municipales, el grupo más perjudicado ha sido el de estudiantes de la educación municipal técnico-profesional, cuya situación no ha sido abordada a la fecha en los estudios del CTA. En sus reportes, el CTA ha omitido de sus análisis esta modalidad educacional, lo cual enmascara el perjuicio a los grupos más vulnerables ${ }^{9}$. Adicionalmente, no han incluido en sus análisis las estimaciones de brechas correspondientes a las pruebas opcionales de Ciencias e Historia y Ciencias Sociales.

A continuación, en la Tabla $\mathrm{N}^{\circ} 1$ se presentan las estimaciones hechas por los autores en relación con el crecimiento de la brecha de rendimiento en todas las PSU. La comparación se realiza entre estudiantes provenientes de establecimientos particulares pagados y aquellos que egresan de la educación técnico-profesional municipal, con los puntajes re-escalados y controlando por nivel socioeconómico. En dicha tabla se presentan los resultados para el grupo total de estudiantes que rinden las pruebas y para el grupo de alumnos que se encuentra en el tercio superior de acuerdo a su rendimiento en la enseñanza media. Este último grupo es muy importante por cuanto los alumnos que se destacan en la enseñanza media son quienes tienen mayores posibilidades de acceder a becas y ayudas económicas, si es que logran ser admitidos en la educación superior.

En el período 2004 a 2009, en las pruebas de Matemática y Lenguaje se aprecia un crecimiento sostenido de la brecha de 22 y 15 puntos respectivamente para el grupo general, siendo el crecimiento de $19 \mathrm{y}$ 15 puntos para el tercio de desempeño superior en la enseñanza media.

${ }^{8}$ Las estimaciones mostradas por el Comité Técnico Asesor (CTA) del Consejo de Rectores en diversas presentaciones son conducentes a error, por cuanto no han estimado en forma apropiada el crecimiento de las brechas. El cambio de escalamiento entre las pruebas las hace incomparables a menos que se las ponga en una misma escala. Al no re-escalar los puntajes correspondientes a las pruebas anteriores a la admisión del 2006 se hace menos evidente el crecimiento de la brecha en las PSU. Asimismo, se requiere homologar los indicadores socioeconómicos de las bases de datos y que varían en el tiempo, lo cual tampoco es considerado en los estudios del CTA.

${ }^{9} \mathrm{Al}$ hacer un ranking del crecimiento de la brecha en función de modalidad y dependencia educacional, las estimaciones arrojan que los grupos más perjudicados son los técnico-profesionales municipales y los técnico-profesionales subvencionados. Dependiendo de la prueba, el perjuicio es mayor para uno u otro grupo. 
TABLA N ${ }^{\circ}$ 1: $\quad$ BRECHAS ESTIMADAS DE RENDIMIENTO POR PRUEBA EXPRESADAS EN PUNTOS PSU ENTRE COLEGIOS PRIVADOS VS. MUNICIPALES TÉCNICOPROFESIONALES CONTROLANDO POR NSE 1,2

\begin{tabular}{|c|c|c|c|c|c|c|c|c|}
\hline \multirow[t]{2}{*}{ Admisión } & \multicolumn{2}{|c|}{ PSU M } & \multicolumn{2}{|c|}{ PSU L } & \multicolumn{2}{|c|}{ PSU C } & \multicolumn{2}{|c|}{ PSU H y CS } \\
\hline & $\begin{array}{l}\text { Total } \\
\text { alums. }\end{array}$ & $\begin{array}{c}\text { Tercil } \\
\text { superior }\end{array}$ & $\begin{array}{l}\text { Total } \\
\text { alums. }\end{array}$ & $\begin{array}{c}\text { Tercil } \\
\text { superior }\end{array}$ & $\begin{array}{l}\text { Total } \\
\text { alums. }\end{array}$ & $\begin{array}{l}\text { Tercil } \\
\text { superior }\end{array}$ & $\begin{array}{l}\text { Total } \\
\text { alums. }\end{array}$ & $\begin{array}{l}\text { Tercil } \\
\text { superior }\end{array}$ \\
\hline 2004 & 65 & 90 & 61 & 80 & 80 & 101 & 53 & 74 \\
\hline 2005 & 72 & 98 & 67 & 88 & 83 & 107 & 58 & 84 \\
\hline 2006 & 79 & 108 & 75 & 99 & 81 & 107 & 58 & 83 \\
\hline 2007 & 81 & 112 & 75 & 97 & 83 & 109 & 62 & 83 \\
\hline 2008 & 86 & 112 & 76 & 98 & 87 & 115 & 65 & 91 \\
\hline 2009 & 87 & 109 & 76 & 95 & 87 & 111 & 70 & 91 \\
\hline $\begin{array}{l}\text { Incremento estimado } \\
\text { período } 2004 \text { a } 2009\end{array}$ & 22 & 19 & 15 & 15 & 7 & 10 & 17 & 17 \\
\hline
\end{tabular}

${ }^{1}$ Control por NSE incluye educación y ocupación de ambos padres e ingreso familiar.

${ }^{2}$ Aun cuando la forma más correcta de presentar las brechas sería corrigiéndolas por la desviación estándar, en aras de la claridad hemos preferido expresarlas directamente en puntos PSU y no en términos de proporción de desviación estándar.

Fuente: Elaboración propia en base a datos provistos por P. Universidad Católica.

Con respecto a la prueba de Ciencias, la brecha parte siendo la más alta de todas las pruebas en el año 2004 (80 y 101 puntos). La brecha en esta PSU también crece en el tiempo, pero en menor grado que la brecha de las pruebas obligatorias. Incluso la versión 2004 - aquella con menores contenidos incluidos - es una prueba que deja fuera de competencia a los alumnos de colegios técnico-profesionales. La explicación más plausible para este fenómeno es que el currículum de esta modalidad no contempla horas en Ciencias Naturales en $3^{\circ}$ y $4^{\circ}$ medio $^{10}$. A pesar de que los alumnos de buen rendimiento escolar provenientes de la modalidad técnico-profesional hagan su mejor esfuerzo en la prueba de Ciencias, la desventaja queda en evidencia cuando se trata de lidiar con quienes han tenido cuatro horas semanales de ciencias durante los dos últimos años de enseñanza media, además de preuniversitarios individuales e institucionales de los colegios con mayores recursos.

${ }^{10}$ Información Ministerio de Educación (Mineduc) en http://www.curriculummineduc.cl/curriculum/planes-de-estudio/media/. 
Con relación a la prueba de Historia y Ciencias Sociales, se advierte a lo largo de los años un fenómeno similar de crecimiento de la brecha que afecta tanto al grupo general como al de alto rendimiento.

A partir de los resultados de la Tabla $\mathrm{N}^{0} 1$ se puede concluir que para todas las PSU la menor brecha en el rendimiento se observa en su versión 2004, aquella que incorporaba menos contenidos temáticos. Los resultados dejan de manifiesto que el aumento de contenidos ha perjudicado al grupo de alumnos provenientes de la educación municipal técnico-profesional. Sin embargo, lo más grave es que el efecto no sólo se aprecia cuando los análisis se hacen para todos los que rinden las PSU, sino que también se evidencia para el grupo de postulantes que se ubica en el tercio superior de rendimiento en la enseñanza media. Cabe señalar que el crecimiento de la brecha en este grupo es particularmente dañino, puesto que afecta al grupo de alumnos vulnerables más capaces dentro de su generación ${ }^{11}$.

El grupo proveniente de la educación técnico-profesional constituye más de un tercio del total de jóvenes que egresan de la enseñanza media y alrededor del $60 \%$ de ellos rinde la PSU, lo que demuestra que esos jóvenes aspiran a cursar la educación superior ${ }^{12}$. Un buen sistema de selección debe ser capaz de detectar a los alumnos talentosos aptos para seguir carreras universitarias, incluyendo a aquellos del segmento técnico-profesional. La comisión a cargo de reformular las pruebas de selección estaba consciente de que las nuevas pruebas eran inadecuadas para la modalidad técnico-profesional por "su referencia a la experiencia curricular de la modalidad Humanista-Científica" y señalaba que las nuevas pruebas tenían "aplicabilidad plena sólo a la matrícula de la modalidad humanista-científica y no a la técnica

${ }^{11} \mathrm{El}$ aumento de la brecha de rendimiento a medida que se han ido incorporando más contenidos en las pruebas es consistente con la baja en el porcentaje de alumnos de colegios municipales que logran ser admitidos en algunas de las universidades más prestigiosas del Consejo de Rectores, tales como las universidades de Chile, Católica, de Concepción, de Santiago y Católica de Valparaíso (Simonsen, 2008).

${ }^{12}$ Cifras preliminares de un estudio que encuestó a estudiantes de último año de enseñanza media técnico-profesional indican que sólo el 9,2\% de los alumnos desea trabajar. La gran mayoría aspira a proseguir estudios superiores. Lamentablemente, tienen mayores restricciones para acceder a programas ofrecidos por universidades que seleccionan en base a la PSU (Ministerio de Educación [Mineduc], "Bases para una Política de Formación Técnico-Profesional en Chile: Informe Ejecutivo”, 2008). 
profesional"13. Sin embargo, los estudiantes de la modalidad técnicoprofesional son evaluados mediante las mismas pruebas que los que egresan de la educación científico-humanista.

A la luz de lo expuesto, cabe preguntarse si se requiere una prueba de máximos contenidos (como es la actual PSU) para seleccionar alumnos a las universidades o si se podrían reducir éstos para promover la equidad entre todos los grupos que la rinden, sin comprometer por ello la capacidad predictiva del instrumento.

\section{3. ¿Predice bien la PSU?}

Ésta es una de las preguntas clave que deben ser respondidas con relación a una prueba de admisión, puesto que si ésta no demuestra tener buena capacidad predictiva, no cumple el objetivo central para el cual fue creada. El CTA del Consejo de Rectores ha entregado tres informes de validez predictiva en los cuales concluye que, a nivel general, la PSU tiene capacidad de predecir el rendimiento universitario ${ }^{14}$. Si bien estos informes presentan falencias metodológicas, en particular al pretender establecer comparaciones con el antiguo sistema PAA $^{15}$, hay en ellos datos reveladores acerca de la capacidad predictiva de la PSU de Matemática y de Lenguaje que merecen ser tomados en consideración y no han sido suficientemente destacados.

Lo primero es que en el caso de la PSU de Matemática el incremento de contenidos no se ha asociado a una mejoría en su capacidad predictiva, sino todo lo contrario. En esta prueba, la mayor capacidad predictiva es la correspondiente a la versión del año 2004, aquella que incorporaba la menor cantidad de contenidos, como queda de manifiesto

${ }^{13}$ Según se señala en el informe de la Comisión Nuevo Currículum de la Enseñanza Media y Pruebas del Sistema de Admisión a la Educación Superior, nov. 22, 2000, pp. 36 y 41 .

14 Documentos técnicos elaborados por el Comité Técnico Asesor (CTA) del Consejo de Rectores (CRUCH): "Estudio Acerca de la Validez Predictiva de los Factores de Selección a las Universidades del Consejo de Rectores" (julio 2006), "Estudio Acerca de la Validez Predictiva de los Factores de Selección a las Universidades del Consejo de Rectores Admisiones 2003 a 2006" (julio 2008) y "Validez Diferencial y Sesgo de Predictividad de las Pruebas de Admisión a las Universidades Chilenas" (septiembre 2010).

${ }^{15}$ Ver M. Koljatic y M. Silva, "Validación de la PSU: Comentarios al 'Estudio Acerca de la Validez Predictiva de los Factores de Selección a las Universidades del Consejo de Rectores"', 2006, pp. 332-346. 
en la Tabla $\mathrm{N}^{\circ}$ 2. Esta misma situación se observa cuando se analizan los resultados por dependencia: la capacidad predictiva de la PSU de Matemática no se ve mejorada por el aumento de contenidos para los colegios particulares pagados, ni para los subvencionados ni para los municipales.

TABLA N ${ }^{\circ}$ 2: CORRELACIÓN PSU MATEMÁTICA CON NOTAS DEL PRIMER AÑO DE CARRERA POR DEPENDENCIA

\begin{tabular}{lccccc}
\hline Admisión & Contenidos* & $\begin{array}{c}\text { Todas las } \\
\text { dependencias }\end{array}$ & $\begin{array}{c}\text { Particulares } \\
\text { pagados }\end{array}$ & $\begin{array}{c}\text { Particulares } \\
\text { subvencionados }\end{array}$ & Municipales \\
\hline 2004 & Reducidos & 0,29 & 0,23 & 0,28 & 0,27 \\
2005 & $\downarrow$ & 0,25 & 0,18 & 0,26 & 0,24 \\
2006 & $\begin{array}{l}\downarrow \\
2007\end{array}$ & 0,24 & 0,20 & 0,22 & 0,24 \\
& Máximos & 0,25 & 0,22 & 0,22 & 0,24 \\
\hline
\end{tabular}

* Flecha indica aumento de contenidos a través de los años.

Fuente de datos: Informe CTA del Consejo de Rectores (2010), "Validez Diferencial y Sesgo de Predictividad de las Pruebas de Admisión a las Universidades Chilenas", septiembre.

Con respecto a la capacidad predictiva de la PSU de Lenguaje, al inspeccionar la información reportada por el CTA se advierten problemas de capacidad predictiva, por cuanto ésta es muy baja a lo largo de los años, incluso cercana a cero y de signo negativo para algunas carreras. Se trata, claramente, de una prueba que no está aportando mayormente a predecir el rendimiento universitario en ninguno de los grupos y que por lo tanto debiera ser revisada ${ }^{16}$.

Y aun cuando en el último informe de validez predictiva del CTA no se da cuenta de la evolución de la capacidad predictiva de las dos pruebas opcionales, la información contenida en uno de los informes

${ }^{16}$ Según se reporta en el último documento técnico del CTA del CRUCH: "Validez Diferencial y Sesgo de Predictividad de las Pruebas de Admisión a las Universidades Chilenas", de septiembre 2010. En la Tabla 6 (p. 22) de dicho informe se reporta que en promedio la correlación de la PSU de Lenguaje con rendimiento universitario es de .08 a .11. En un informe anterior, del año 2008 y que incluye datos relativos a las admisiones 2003 a 2006, se aprecia que la PSU de Lenguaje no sólo presenta correlaciones cercanas a cero para muchas carreras sino que para algunas la correlación entre los puntajes de la PSU y el rendimiento universitario, aunque de baja magnitud, es de signo negativo. Una correlación de signo negativo es contraria a lo esperado, por cuanto indicaría que a mayor puntaje en la prueba, menor sería el rendimiento en el primer año de universidad. 
previos (2008) permite afirmar que al igual que en el caso de la prueba de Matemática, la capacidad predictiva de las pruebas de Ciencias y de Historia y Ciencias Sociales no mejora con el aumento de conte$\operatorname{nidos}^{17}$.

\section{Pruebas de admisión: selección con equidad}

Hasta hace algunas décadas, la calidad de una prueba de selección se medía exclusivamente sobre la base de una simple correlación: si los resultados de la prueba servían para predecir el rendimiento universitario, la prueba era "buena". Sin embargo, hoy en día la predictibilidad es una condición necesaria, pero no suficiente, para avalar el uso legítimo de un instrumento para fines de selección ${ }^{18}$.

Las consideraciones de equidad han cobrado relevancia al momento de emitir un juicio acerca de la idoneidad de un instrumento para propósitos de selección. A modo de ejemplo, aun cuando una prueba de inglés predijera muy bien el rendimiento universitario, su uso sería cuestionable en un país en que la enseñanza de un segundo idioma fuera privilegio de una elite, por la ventaja que daría a dicho grupo aun cuando la prueba se circunscribiera a aquellos contenidos curriculares mínimos para todas las escuelas. Lo recomendable sería escoger otro tipo de prueba de admisión que posibilitara la detección de alumnos idóneos y que perjudicara lo menos posible a los grupos vulnerables de la población.

En la actualidad, la legitimidad en el uso de pruebas de admisión como mecanismos de selección a la educación superior está dada por dos criterios técnicos: la equidad para todos los subgrupos de la pobla-

${ }^{17}$ La capacidad predictiva para la PSU de Ciencias para el 2004 y el 2006 es de .26 y .24 , respectivamente. La de Historia y Ciencias Sociales es de .08 y .06 para el mismo período. En esta última se advierte, al igual que en la prueba de Lenguaje, una baja capacidad predictiva relativa a las pruebas de Matemática y Ciencias. El último informe de "Validez Diferencial y Sesgo de Predictividad de las Pruebas de Admisión a las Uiversidades Chilenas" (CTA del Consejo de Rectores, septiembre 2010) no hace referencia alguna a las pruebas optativas, por lo cual no hay antecedentes acerca de su capacidad predictiva para los grupos que la rinden. Llama la atención que el informe se explaya en la predicción diferencial por género, omitiendo análisis relevantes para evaluar su funcionamiento en los grupos más vulnerables según modalidad y dependencia educacional.

${ }^{18}$ L. Shepard, "Evaluating Test Validity”, 1993, pp. 405-450. 
ción y el grado en el cual los puntajes en las pruebas predicen el rendimiento universitario para los distintos subgrupos de la población ${ }^{19}$.

Si la PSU del año 2004, aquella con menores contenidos, tiene tan buena o mejor capacidad predictiva que las de los años subsiguientes, ¿por qué no considerar una prueba de menores contenidos curriculares para efectos de admisión si ello no compromete su capacidad predictiva y en cambio favorece la equidad?

Para entender la decisión de incorporar el máximo posible de contenidos en las pruebas, aun cuando éstos no sean necesarios para una mejor predicción en la mayoría de las carreras, es necesario entender la lógica que inspiró el cambio del sistema PAA por el sistema $\mathrm{PSU}^{20}$.

\section{Inclusión del máximo de contenidos}

Aun cuando la capacidad predictiva de las pruebas es de capital importancia, el cambio del sistema de PAA por la PSU no buscaba mejorar la capacidad predictiva de las pruebas. Al respecto, la Comisión Nuevo Currículum señalaba expresamente en el documento de 2000 a favor del cambio de pruebas: "En términos de su validez predictiva y de constructo, las pruebas del sistema de admisión [PAA] exhiben estándares adecuados y que no distan de los que se logran con este tipo de pruebas en otros países. No residen aquí, es decir, en sus características técnicas como instrumentos de medición, los problemas o limitaciones que llaman a readecuarlas" (p. 38).

Cabe recordar que Chile, a fines de los 90, se encontraba embarcado en una reforma de la educación media. En ese contexto se tomó la decisión de cambiar las pruebas para alinear los contenidos curriculares de la reforma educacional con las pruebas de admisión, con miras a garantizar la sustentabilidad de la reforma de la educación media. Las nuevas pruebas, alineadas con el nuevo currículum, servirían tanto para fines de admisión como de evaluación de la enseñanza media.

${ }^{19}$ V. Santelices y M. Wilson, "Unfair Treatment?: The Case of Freedle, the SAT and the Standardization Approach to Differential Item Functioning", 2010, pp. 106-133.

${ }^{20}$ Una motivación para el cambio de pruebas fue la de evaluar la enseñanza media y garantizar la sustentatibilidad de la reforma educacional. El doble propósito que cumplen las nuevas pruebas está señalado en documentos del Mineduc, "Comisión Nuevo Currículum de la Enseñanza Media y Pruebas en el Sistema de Admisión a la Educación Superior", 2000, noviembre 22, y del Banco Mundial, "Implementation Completion Report on a Loan in the Amount of U.S.\$35 Million to the Republic of Chile for a CLSecondary Education", 2001, octubre 19. Para evaluar la enseñanza media, el mecanismo adecuado era un Simce de $4^{\circ}$ medio. 
El problema surgió por un error conceptual: diseñar las pruebas a partir del currículum se entendió, erróneamente, como sinónimo de tener que incluir en éstas el 100\% de los vastos "contenidos mínimos" prescritos para la enseñanza media. Este error fue el que comprometió las oportunidades de acceso universitario de los grupos más vulnerables de la población al estar expuestos a una mala calidad de educación donde las materias evaluadas en las pruebas no son enseñadas. En lugar de seleccionar los contenidos relevantes para las distintas carreras y diseñar las pruebas en función de su medición, se optó por la inclusión universal de contenidos, fueran éstos importantes o no para predecir el éxito en los distintos programas de educación superior.

Si tomamos como ejemplo el caso de Matemática, el error es evidente, ya que los requisitos de conocimientos en esta área son muy distintos para quien desea estudiar Ingeniería Civil que para quien desea estudiar Derecho. Sin embargo, la prueba para acceder a ambas es exactamente la misma. No se puede esperar que la misma prueba de Matemática haga un buen trabajo de selección en todas las carreras, como ha sido señalado por connotados académicos ${ }^{21}$.

La reformulación de las pruebas de admisión debió centrarse en seleccionar aquellos contenidos curriculares relevantes para tener éxito en la universidad. En Chile, por la forma en que está organizado el sistema universitario, los alumnos entran directamente a carreras profesionales que requieren de distintos niveles de conocimientos y competencias. La situación es muy distinta en el caso de países en los cuales los alumnos entran a un college en que se les entrega formación general y luego se opta por carreras profesionales. En Chile se habría justificado ampliamente desarrollar distintas pruebas de matemáticas — una de contenidos avanzados y otra de contenidos más básicos - para efectos de seleccionar alumnos a las distintas carreras. Hay carreras en que la pérdida de las antiguas pruebas de conocimientos específicos (PCE) se tradujo en una baja significativa de la capacidad predictiva de las pruebas de ingreso a la universidad. Es el caso de la carrera de Ingeniería Civil de la Univer-

${ }^{21}$ Ello queda bien representado en las palabras de Rafael Benguria, Premio Nacional de Ciencias y profesor de Física y Matemáticas de la UC: "La PSU de matemáticas es muy elemental, se satura, apela mucho a la memoria [...] El año pasado me llegaron alumnos muy afligidos, contándome que fueron puntaje nacional en la PSU de matemáticas y que habían reprobado todos los cursos [...]". Véase E. Irarrázabal, "El Teorema de Benguria”, El Mercurio, 4/9/2005. 
sidad Católica, donde se perdió casi el 7\% de capacidad predictiva con el cambio del sistema de PAA y PCE al sistema PSU ${ }^{22}$.

Por otra parte, la prueba de Lenguaje no sólo tiene déficits en su capacidad predictiva, como se ha señalado, sino que ha sido criticada en función de sus contenidos por connotados educadores y académicos ${ }^{23}$. $\mathrm{Si}$ a lo anterior se añade la brecha creciente en el rendimiento que favorece a los alumnos provenientes de colegios particulares, resulta imperativo reformularla.

Ante esta realidad, la decisión acerca de cuáles contenidos mantener en las pruebas requerirá de un análisis serio y riguroso, consultando a académicos de las distintas áreas cuál es la línea base de conocimientos que necesitan tener los alumnos para sortear con éxito las exigencias del primer año de universidad en distintas carreras ${ }^{24}$.

\section{EI uso de las PSU para otorgar beneficios y becas}

A la luz de las falencias de las actuales pruebas de admisión en cuanto a su capacidad predictiva y a la equidad, también debiera revisarse el énfasis que se les ha dado a las PSU de Lenguaje y de Matemática en la asignación de beneficios y becas ${ }^{25}$.

Por ejemplo, recientemente se estipuló que todo alumno que obtenga 600 puntos promedio o más en las PSU de Matemática y Lenguaje será beneficiario de una beca de estudios si opta por estudiar Pedago-

22 S. Prado, "Admission of Chilean Universities. The Case of Engineering", 2010, trabajo presentado en la Decimotercera Conferencia organizada por la Agencia Nacional para la Educación Superior de Suecia. Universidad de Umea, Suecia, junio 15-17.

${ }^{23}$ Entre ellos, los profesores Diego Ibáñez Langlois y Hugo Montes, Premio Nacional de Educación.

${ }^{24}$ Asimismo, debe revisarse la racionalidad de entregar un único puntaje de ciencias independientemente de la prueba opcional que se rinda. Es evidente que para la carrera de Ingeniería sería mucho más razonable que los postulantes rindieran el módulo opcional de Física en vez del de Biología.

${ }^{25}$ A lo largo de los años de aplicación de la PSU se han definido puntos de corte arbitrarios para la admisión de alumnos a las universidades del Consejo de Rectores y para asignar ayudas estudiantiles, sin atender a consideraciones técnicas. El legítimo uso de puntos de corte requiere de numerosos estudios que los validen. Aun cuando ello se cumpla, la asignación de beneficios no debe recaer exclusivamente en los puntajes de las pruebas, como señala una de las más prestigiosas organizaciones de EE. UU., orientada a promover altos estándares éticos en los procesos de admisión (National Association for College Admission Counseling, NACAC, 2009. Accesible en: http://www.nacacnet.org/ AboutNACAC/Policies/Documents/SPGP.pdf). 
gía $^{26}$. Si bien desde un punto de vista de la transparencia es muy positivo usar mecanismos objetivos en la asignación de beneficios, conviene revisar si es adecuado usar exclusivamente los puntajes de las PSU de Lenguaje y Matemática para este propósito, si se considera que la PSU de Lenguaje parece ser un muy mal predictor del éxito en carreras de Pedagogía, según lo revelan datos emanados de informes oficiales del CTA del Consejo de Rectores y que se indican en la Tabla $\mathrm{N}^{\circ} 3$. TABLA N ${ }^{\circ}$ 3: $\quad$ CORRELACIÓN PROMEDIO PONDERADO ACUMULADO DEL PRIMER AÑO
CON FACTORES DE SELECCIÓN (ADMISIÓN 2006)

NEM PSU L PSU M PSU Cs PSU H

Ped. Gral. Bás./UC (n=118)

Educación Párvulos/UC ( $\mathrm{n}=116)$

Ped. Inglés/UC Temuco $(\mathrm{n}=90)$

Lic. en Ed. y Ped. Bás./UMCE $(\mathrm{n}=88)$

Ped. Gral. Básica/UCSSC $(n=87)$

Lic. en Educ. y Parvularia/UMCE $(n=77)$

Ped. en Cs./UC Maule ( $\mathrm{n}=77$ )

Ped. en Ed. Gral. Básica/UBiobío ( $\mathrm{n}=76$ )

Ped. Gral. Bás. Especializ./UC Temuco $(n=76)$

$\begin{array}{rrrrr}.28 & -.02 & . \mathbf{2 9} & .02 & .06 \\ .06 & -.03 & .05 & -.17 & -.02 \\ .01 & . \mathbf{3 3} & . \mathbf{3 3} & & .20 \\ .09 & .04 & . \mathbf{2 1} & & .05 \\ \mathbf{1 3} & -.03 & .11 & & .00 \\ \mathbf{. 1 6} & -.07 & -.07 & & -.03 \\ . \mathbf{2 4} & .09 & .22 & .07 & \\ \mathbf{4 6} & -.15 & .03 & & -.06 \\ \mathbf{4 3} & .19 & .15 & & -.10\end{array}$

Nota: Se destaca en negritas el predictor con más alta correlación con el promedio ponderado acumulado del primer año de la carrera.

Fuente: Comité Técnico Asesor (CTA) del Consejo de Rectores de las Universidades, "Estudio acerca de la Validez Predictiva de los Factores de Selección a las Universidades del Consejo de Rectores, Admisiones 2003 a 2006", julio 2008. Incluye todas las carreras de Pedagogía reportadas en el informe para el año 2006, por ser la prueba más semejante a la que se aplica hoy en día (considerando la cantidad de contenidos evaluados).

Si bien hay una amplia variabilidad en la capacidad predictiva de los distintos indicadores que se presentan en la Tabla $\mathrm{N}^{\mathrm{o}} 3$, se aprecia que en cinco de las nueve carreras de Pedagogía que reporta el informe del CTA, el mejor predictor de rendimiento en el primer año de carrera es el promedio de notas de enseñanza media. Para tres de ellas el mejor predictor es la PSU de Matemática y en una de ellas es la PSU de Cien-

${ }^{26}$ Se trata de las nuevas becas "Vocación de Profesor" que se implementarán a partir de la admisión 2011. Detalles en www.becavocaciondeprofesor.cl. 
cias, aunque con signo contrario al esperado ${ }^{27}$. La PSU de Lenguaje y las PSU opcionales se perfilan como los peores predictores para esta muestra de carreras, con indicadores cercanos a cero. Para efectos de la selección en estas carreras de Pedagogía, un criterio únicamente basado en un promedio de las PSU no garantizaría necesariamente la selección de alumnos exitosos en el primer año de carrera. En particular, la exclusión de las notas de enseñanza media y la inclusión de la prueba de Lenguaje como factor de selección para las becas no parecen tener asidero ni desde un punto de vista de la capacidad predictiva ni tampoco desde la perspectiva de la equidad.

En relación a la equidad, la Tabla $\mathrm{N}^{\circ} 4$ muestra el ejercicio de cómo variaría la composición por dependencia de los potenciales beneficiarios de las becas (si se mantuviera el criterio de los 600 puntos) frente a distintas combinaciones de predictores.

TABLA N ${ }^{\circ}$ 4: $\quad$ PORCENTAJES DE BENEFICIARIOS POTENCIALES PARA COMBINACIONES DE DISTINTOS PREDICTORES

\begin{tabular}{lccccc}
\hline Dependencia & Sólo NEM & Sólo PSU M & $\begin{array}{c}\text { Promedio } \\
\text { PSU L } \\
\text { ySU M }\end{array}$ & $\begin{array}{c}\text { Promedio } \\
\text { PSU M } \\
\text { y NEM }\end{array}$ & $\begin{array}{c}\text { Prom. PSU M, } \\
\text { PSU L } \\
\text { y NEM }\end{array}$ \\
\hline Part. pagado & $17 \%$ & $34 \%$ & $35 \%$ & $27 \%$ & $31 \%$ \\
Part. subv. & $45 \%$ & $42 \%$ & $42 \%$ & $45 \%$ & $44 \%$ \\
Municipal & $38 \%$ & $24 \%$ & $23 \%$ & $28 \%$ & $25 \%$ \\
N total elegible & $(\mathrm{n}=65.219)$ & $(\mathrm{n}=44.234)$ & $(\mathrm{n}=41.930)$ & $(\mathrm{n}=48.367)$ & $(\mathrm{n}=43.773)$
\end{tabular}

Fuente: Elaboración propia en base a datos para la admisión 2009, para puntajes mayores o iguales a 600 puntos, proporcionados por la P. Universidad Católica de Chile.

Según se puede apreciar en la Tabla $\mathrm{N}^{0} 4$, el promedio de las PSU de Lenguaje y Matemática es la combinación que más restringe el número total de potenciales beneficiarios de las becas $(\mathrm{N}=41.930)$. Al combinar el puntaje asociado a notas de enseñanza media y el puntaje PSU de Matemática (esto es, excluyendo la PSU de Lenguaje), se obtiene un número potencial de becarios mayor que el anterior y que

${ }^{27}$ Naturalmente, en este caso se esperaría que las correlaciones fueran altas y de signo positivo. 
asciende a 48.367. Adicionalmente, con esta combinación se aumenta significativamente el porcentaje de potenciales becarios provenientes del sector municipal: de 23\% con el promedio de PSU de Lenguaje y Matemática, se incrementa a $28 \%$, aumento que corresponde a 5 puntos porcentuales $^{28}$.

En resumen, cabe reiterar que para fines de escoger alumnos que tengan éxito en la carrera de Pedagogía y que se conviertan a futuro en maestros destacados, sería deseable realizar un estudio más acabado de la capacidad predictiva de la PSU y notas de enseñanza media para todas las carreras de Pedagogía del sistema universitario. Se ve que hay una variabilidad sustancial en términos de la capacidad predictiva de las pruebas para distintas universidades. Asimismo es necesario investigar el efecto de la inclusión de otras variables que, además de promover un acceso más equitativo a los beneficios, pueden ser buenos predictores del rendimiento universitario en Pedagogía, como, por ejemplo, la posición relativa que ocupa el alumno en su grupo curso, entre otras ${ }^{29}$.

\section{Aspectos positivos asociados al cambio y sugerencias}

Hasta aquí nos hemos referido a los problemas que presentan las pruebas. Ahora resaltaremos algunos corolarios positivos que se han revelado en los últimos siete años y que se asocian al cambio de pruebas

${ }^{28}$ Consistente con los hallazgos del crecimiento de las brechas entre colegios particulares y municipales, al repetir el ejercicio empleando los datos de la admisión 2004, se advierte que con pruebas de menores contenidos hay un porcentaje mayor de alumnos de colegios municipales en condiciones de recibir el beneficio. Por ejemplo, al usar el criterio de 600 puntos promedio en la PSU de Lenguaje y Matemática, el porcentaje de alumnos de establecimientos municipales en condición de postular a la beca de Pedagogía en lugar de ser el $23 \%$ sería el $26 \%$ si se empleara la prueba que evalúa menos contenidos.

${ }^{29}$ A futuro, sería conveniente que las becas "Vocación de Profesor" no sólo fueran asignadas sobre la base del puntaje en la PSU como único criterio. Además de incluir otros indicadores de rendimiento en la enseñanza media, habría que considerar variables de otra naturaleza. Por ejemplo, en este caso se justificaría ampliamente buscar mecanismos complementarios para detectar a quienes tienen vocación pedagógica. Tanto o más importantes que los conocimientos al entrar a la carrera (que es lo que mide la PSU) es cuán motivados están para dedicar su vida laboral a la educación. Asimismo, es más lógico evaluar el caudal de conocimientos al egreso de la carrera que al inicio, pues lo que importa es garantizar que se ha alcanzado un dominio satisfactorio de los contenidos que se deberá enseñar. 
de admisión y, finalmente, propondremos algunas medidas para el mejoramiento del sistema.

Dentro de los aspectos positivos asociados al cambio está la atención que ha concitado la brecha creciente de rendimiento y que se verifica año a año entre los postulantes de la educación privada y la educación municipal. Antes del cambio en las pruebas, el tema de la disparidad de rendimiento entre los grupos con mayores recursos y los más vulnerables no era un foco de atención. Fueron los creadores de las nuevas pruebas quienes pusieron de relieve el tema de la equidad en relación con las pruebas de admisión. Si bien las expectativas de un mayor espacio de equidad no se cumplieron con el cambio de pruebas, la sensibilidad hacia el tema en la esfera pública es un aspecto positivo que puede contribuir a abrir espacios de equidad reales en el sistema de admisión.

Otro aspecto positivo del cambio de pruebas es que ha favorecido la profesionalización de la medición educacional en el ámbito universitario. El Departamento de Medición y Registro Educacional (DEMRE), entidad a cargo de la elaboración y aplicación de las pruebas de selección, opera mejor hoy que ayer gracias al entrenamiento recibido por parte del Educational Testing Service (ETS) ${ }^{30}$ en los años transcurridos luego del cambio de pruebas. En este lapso, con la entrada de otros competidores que pueden prestar servicios en esta área (como el Mide $\mathrm{UC}$, entidad que apoya la realización de proyectos de investigación educacional), el DEMRE ya no constituye un monopolio en el ámbito de las pruebas de admisión. La mayor oferta de servicios en esta área constituye un aporte para el desarrollo de la medición en el país.

La experiencia del cambio y sus errores también ha tenido su repercusión positiva en el impulso que se ha dado a la búsqueda de mecanismos alternativos de acceso. Así, la Universidad de Santiago ha sido pionera en la implementación de un sistema de admisión paralelo a la PSU (Propedéutico USACH-Unesco) con miras a gestar un espacio de equidad para quienes, teniendo la capacidad de aprender, han quedado en abierta desventaja para acceder a las universidades tradicionales por no haber tenido las oportunidades de aprender.

Otro aspecto positivo asociado al cambio se manifiesta en la cautela con que se ha enfrentado el desarrollo y evaluación de instrumentos complementarios para ser utilizados en la admisión universitaria. Así,

${ }^{30}$ ETS es la entidad a cargo de la elaboración de las pruebas de admisión universitaria SAT en los Estados Unidos. 
los resultados de la evaluación piloto del proyecto de la Universidad Católica de Chile iniciado en el año 2007, "Instrumentos Complementarios de Admisión Universitaria", se esperan para mediados del año 2011. Los nuevos instrumentos consisten en un formulario de postulación ampliado (en el que los alumnos reportan, entre otros, las actividades extracurriculares desarrolladas durante la enseñanza media y los premios académicos y no académicos obtenidos hasta entonces), un ensayo de pensamiento crítico, una reflexión personal y tres cuestionarios estandarizados para medir habilidades metacognitivas, habilidades sociales y proactividad. El proyecto se ha perfilado como un "potencial aporte en términos de menores brechas en la admisión a las universidades chilenas" ${ }^{\prime 1}$. Si bien el anexar este tipo de instrumento conlleva riesgos ya que incorpora un elemento de discrecionalidad en las decisiones de admisión, habría que poner en la balanza la ganancia potencial de su uso en materia de equidad.

En el marco de la mayor sensibilidad hacia temas de equidad e inclusión, también ha cobrado fuerza la opción del ranking o el puesto que ocupa un alumno en su grupo curso como un elemento adicional a considerar en el marco de la admisión universitaria. Si bien hay quienes han abogado por años para que este indicador se incluya con el fin de mejorar la capacidad predictiva, hoy quienes apoyan la inclusión del ranking valoran también la ventaja de éste en pro de la equidad, ya que bonificaría a todos los alumnos que se han destacado sobre sus compañeros y han demostrado haber aprovechado de mejor manera las oportunidades de aprendizaje que les ofrece su ambiente escolar. Con este indicador no hay brecha de equidad y tiene la ventaja de que es de costo cero, a diferencia de otros mecanismos de admisión complementarios basados en la aplicación de cuestionarios y entrevistas que son costosos de administrar.

No obstante los anteriores aspectos positivos, hay cambios urgentes que implementar en la PSU. Con relación a lo que resta por mejorar, luego de siete años de aplicación, se espera que se concrete una auditoría independiente y transparente de la calidad de las pruebas a la luz de los propósitos para los cuales se están empleando los puntajes. La auditoría a la PSU debería responder preguntas básicas con respecto

${ }^{31} \mathrm{http}: / /$ www.uc.cl/webpuc/piloto/p_estudiantes.html. 
a la calidad y equidad de las pruebas para efectos de selección en el contexto de Chile.

La revisión de la PSU debería contemplar un análisis acabado no sólo de lo que en la actualidad miden las pruebas sino de lo que deberían medir. La inclusión de todos los contenidos curriculares, independientemente de la carrera a seguir, fue una mala decisión que debe ser corregida, ya que el aumento de contenidos ha incrementado la situación de desventaja para los grupos más vulnerables de la población.

Es un hecho que, aun controlando por nivel socioeconómico, mientras mayor cantidad de contenidos se incorporan a la evaluación, mayor es la desventaja que enfrentan los grupos más desposeídos de la población. Con la totalidad de los contenidos mínimos de la enseñanza media incluidos en la PSU, ésta se ha ido convirtiendo cada vez más en un instrumento que mide oportunidad de aprender en lugar de medir capacidad de aprender.

$\mathrm{Ni}$ aun el grupo de mejor rendimiento en la enseñanza media se ha escapado del perjuicio asociado al aumento de contenidos. El efecto puede parecer marginal, pero no lo es. Las universidades más prestigiosas del sistema chileno han visto año a año caer los porcentajes de alumnos de colegios municipales que logran acceder a ellas a partir de la implementación de la $\mathrm{PSU}^{32}$. El aumento de contenidos en las pruebas no se sostiene ni en función de un argumento de mayor equidad ni sobre la base de una ganancia en la capacidad predictiva de las pruebas: la capacidad predictiva de las PSU no mejora en absoluto agregando a éstas más contenidos.

Finalmente, el mayor beneficio que podría extraerse de la experiencia vivida con el cambio de pruebas es que las autoridades tomen conciencia de la necesidad de evaluar externamente y en forma periódica todas aquellas pruebas que tienen altas consecuencias individuales, como la PSU, la evaluación docente y la prueba INICIA. No debería existir ninguna prueba de altas consecuencias que no cuente con una auditoría periódica, realizada por expertos internacionales, independientes y altamente calificados, para garantizar su calidad y el correcto uso de sus resultados. En el caso de Chile, en que el acceso a la educación universitaria es clave para la movilidad social, es fundamental que el ministerio designe un comité permanente que lo asesore de manera que

32 E. Simonsen, "Ingreso de Alumnos Municipales a Universidades Top Baja $10 \%$ en 6 Años", 2008. 
las pruebas de admisión sean instrumentos que favorezcan la equidad de oportunidades para todos los jóvenes. El rol del ministerio no sólo debe ser el de financiar el sistema a través de las becas PSU, como lo hace en la actualidad, sino servir de garante de que el sistema de pruebas cumple con los estándares de calidad y equidad que debe exigirse a un sistema de selección universitaria.

\section{REFERENCIAS}

Banco Mundial (2001). "Implementation Completion Report on a Loan in the Amount of U.S.\$35 Million to the Republic of Chile for a CL-Secondary Education". Octubre 19.

Beyer, H. (2009). "Igualdad de Oportunidades y Selección a las Universidades". Puntos de Referencia, $N^{\circ}$ 303, Centro de Estudios Públicos.

Bravo, D. Declaraciones en entrevista (2002). Diario Austral 21/7/2002.

Bravo, D. y otros (2000). "Reformulación de las Pruebas de Selección a la Educación Superior". Proyecto Fondef. Santiago, Chile.

Comisión Nuevo Currículum de la Enseñanza Media y Pruebas del Sistema de Admisión a la Educación Superior: Informe sometido en consulta previa a la Ministra de Educación (2000). Manuscrito no publicado. Nov. 22, 2000.

Comité Técnico Asesor (CTA) del Consejo de Rectores (2006). "Estudio Acerca de la Validez Predictiva de los Factores de Selección a las Universidades del Consejo de Rectores". Julio.

- (2008). "Estudio Acerca de la Validez Predictiva de los Factores de Selección a las Universidades del Consejo de Rectores Admisiones 2003 a 2006". Julio.

- (2010). "Validez Diferencial y Sesgo de Predictividad de las Pruebas de Admisión a las Universidades Chilenas". Septiembre.

Eyzaguirre, B. y C. Le Foulon (2002). "El SIES: Un Proyecto Prematuro". Estudios Públicos, $\mathrm{N}^{\circ} 87$.

Fontaine, A. (2002). "Peligro en el SIES". Estudios Públicos, № 86.

Irarrázabal, E. (2005). "El Teorema de Benguria”. El Mercurio, 4/9/2005.

Koljatic, M. y M. Silva (2006). "Validación de la PSU: Comentarios al 'Estudio Acerca de la Validez Predictiva de los Factores de Selección a las Universidades del Consejo de Rectores"'. Estudios Públicos, 104.

Koljatic, M., M. Silva y R. Cofré (2010). "Achievement Versus Aptitude in College Admissions: A Cautionary Note Based on Evidence from Chile". Trabajo presentado en la Decimotercera Conferencia organizada por la Agencia National para la Educación Superior de Suecia. Universidad de Umea, Suecia, junio 15-17.

Ministerio de Educación (Mineduc), Gobierno de Chile. http://www.curriculummineduc.cl/curriculum/planes-de-estudio/media. (2008). "Bases para una Política de Formación Técnico-Profesional en Chile: Informe Ejecutivo". 
Unidad de Currículum y Evaluación (mayo 2009). Resultados Nacionales Simce 2008. Accesado julio 2010: http://www.simce.cl/fileadmin/Documentos_y_ archivos_SIMCE/Informes_Resultados_2008/Informe_Nacional_2008.pdf

National Association for College Admission Counseling. 2009. Statements of Principles of Good Practice. Agosto 26. Accesible en: http://www.nacacnet.org/ AboutNACAC/Policies/Documents/SPGP.pdf

OECD (2009). Reviews of National Policies for Education: Tertiary Education in Chile Paris: OECD.

Prado, S. (2010). "Admission in Chilean Universities. The Case of Engineering". Trabajo presentado en la Decimotercera Conferencia organizada por la Agencia Nacional para la Educación Superior de Suecia. Universidad de Umea, Suecia, junio 15-17.

Santelices, V. \& M. Wilson (2010). "Unfair Treatment?: The Case of Freedle, the SAT and the Standarization Approach of Differential Item Functioning". Harvard Educational Review, 80(1), 106-133.

Shepard, L. (1993). "Evaluating Test Validity". Review of Educational Research, 405-450.

SIES: Un Desafío para la Enseñanza. Portal EducarChile 26/4/2002. Accesible en: http:// www.educarchile.cl/Portal.Base/Web/VerContenido.aspx?ID=73956.

Simonsen, E. (2008). "Ingreso de Alumnos Municipales a Universidades Top Baja 10\% en 6 Años". La Tercera, 30 de noviembre, p. 40.

Universidad Católica de Chile. "Instrumentos Complementarios para la Admisión Universitaria”". http://www.uc.cl/webpuc/piloto/p_estudiantes.html. 